\title{
Two novel cross-protective antigens for bovine Pasteurella multocida
}

\author{
HUIHUI DU ${ }^{1,2^{*}}$, CHENLU WU ${ }^{2 *}$, CHUNMING LI $^{2}$, RENDONG FANG ${ }^{2}$, JIANWEI MA ${ }^{2}$, \\ JIALE JI $^{2}$, ZHIHONG LI ${ }^{1}$, NENGZHANG LI ${ }^{2}$, YUANYI PENG ${ }^{2}$ and ZEYANG ZHOU ${ }^{1}$ \\ ${ }^{1}$ The State Key Laboratory of Silkworm Genome Biology, ${ }^{2}$ College of Animal Science \\ and Technology, Southwest University, Chongqing 400716, P.R. China
}

Received February 11, 2016; Accepted February 20, 2017

DOI: $10.3892 / \mathrm{mmr} .2017 .7153$

\begin{abstract}
Pasteurella multocida is an important pathogen that leads to a range of diseases that have severe economic consequences on cattle production. In order to develop an effective cross-protective component vaccine, an immunoproteomics approach was used to analyze outer membrane proteins (OMPs) of the P. multocida serotype A, B and F strains. Candidate antigen molecules from the whole genome were screened via linear trap quadrupole mass spectrometry and bioinformatics analysis, and the reactogenicity of the candidate antigen molecules was analyzed via cloning, expression, and ELISA or protein immunoblotting, and the vaccine efficacy of the candidate molecules was determined in infective animal models and cross-protective antigens may be obtained. rPmCQ2_2g0128, rPmCQ2_1g0327 and rPmCQ2_1g0020 proteins were selected. Their protective rates were 40/30/20\% (rPmCQ2_2g0128), 50/40/0\% (rPmCQ2_1g0327) and 0/40/30\% (rPmCQ2_1g0020) against ten-fold median lethal dose $\left(10 \mathrm{LD}_{50}\right)$ of the $P$. multocida serotypes $\mathrm{A}, \mathrm{B}$ and $\mathrm{F}$ in a mouse model, respectively. The results suggested that the two proteins rPmCQ2_2g0128 and rPmCQ2_1g0327 may be used as vaccine candidates against bovine $P$. multocida serotypes A, B. To the best of our knowledge, the present study was the first to identify cross-protective antigens, extracted OMPs from bovine $P$. multocida.
\end{abstract}

Correspondence to: Professor Zeyang Zhou, The State Key Laboratory of Silkworm Genome Biology, Southwest University, 2 Tiansheng Road, Beibei, Chongqing 400716, P.R. China

E-mail: zyzhou@swu.edu.cn

Professor Yuanyi Peng, College of Animal Science and Technology, Southwest University, 2 Tiansheng Road, Beibei, Chongqing 400716, P.R. China

E-mail: pyy2002@sina.com

${ }^{*}$ Contributed equally

Key words: cross-protective antigens, bovine Pasteurella multocida, outer membrane protein

\section{Introduction}

Pasteurella multocida (P. multocida) is an important opportunistic pathogen that leads to a range of diseases in domestic and wild animals, including cattle (1), pigs (2), rabbits (3) and birds (4). $P$. multocida strains are classified into five serotypes: A, B, D, E and F, based on the capsular composition and 1-16 somatic serovars $(5,6)$. The $P$. multocida serotypes $\mathrm{A}, \mathrm{B}$ and $\mathrm{F}$ are the primary serotypes responsible for bovine pneumonia and hemorrhagic septicemia, and abscesses, otitis and septicemia in rabbits (7-9). In addition, the $P$. multocida serotype A:1 (Pm A:1) is associated with fatal pneumonia and septicemia in cattle and buffaloes (10), which are responsible for $30 \%$ of cattle deaths worldwide and $\$ 1$ billion in annual loss for beef cattle production in North America (11).

Vaccinations with inactivated bacteria (bacterins) or live attenuated bacteria is an effective and economic method for improving the condition of and protecting animals against $P$. multocida infections (11). However, $P$. multocida bacterins provide limited protection for heterologous serotype and the live/attenuated vaccines may revert to virulence, thus infecting animals (8). Therefore, the present study focused on P. multocida immunogens that elicit cross-protective immunity and may be used in vaccines against pasteurellosis (12). Outer membrane proteins (OMPs) provide protective immunity against $P$. multocida infection (13); therefore, OMPs may be primary protective antigens. Therefore, the $P$. multocida outer membrane proteome may aid in identifying potential vaccine candidates and diagnostic antigens. A high immunogenicity lipoprotein in P. multocida, PlpE, provided $80-100 \%$ protection in mice and induced cross-serotype protective immunity (14). The recombinant adhesive protein $\mathrm{rCp} 39$ provided high levels of protection from fowl cholera in chickens via challenge-exposure with the parental strain P-1059 or the heterologous strain $\mathrm{X}-73$ (serotype A:1) compared with various other vaccines, e.g., PlpB and MOMPs (15). Iron-regulated OMPs (IROMPs) provided protection against $P$. multocida in rabbits (16) and calves $(17,18)$. The recombinant $\mathrm{OmpH}$-based vaccine provided cross-protection against avian P. multocida strains (15). These recombinant proteins may be used as subunit vaccines, which are safe and efficient for immuno-compromised animals; however, they provide limited protection against heterologous serotypes. Therefore, identifying cross-protective antigens 
may improve current vaccines. The present study was based on an immunoproteomic approach and identified two novel immunogenic OMPs with evident cross-protection against bovine $P$. multocida A, B and F strains. These two immunogenic OMPs may be promising candidates for a universal cross-protective vaccine that may have effective antigen variants. To the best of our knowledge, the present study was the first to identify cross-protective antigens extracted from OMPs in bovine $P$. multocida.

\section{Materials and methods}

Bacterial strains and culture conditions, vectors, and primers. The bovine $P$. multocida serotype A PmCQ2 and PmCQ6 strains were isolated from lungs of feedlot calves suffering from fatal pneumonia at several cattle farms in Chongqing, China between May 2012 and December 2013. The bovine P. multocida serotype F PmF strain was isolated from lungs of feedlot calves suffering from disease of respiratory system at Gaojiazhen farms in Fengdu (Chongqing, China, longitude/latitude 107.70/29.89) in October 2013. The serotype B strain PmB is a well-characterized pathogenic strain and was purchased from the Chinese Veterinary Drug Supervision (cat. no. CVCC450; Beijing, China). The animal studies were performed with approval from the ethics committee of Southwest University (Beibei, China; permit no. 11-1025) and in compliance with the Laboratory Animal Care principles of the National Institutes of Health (Bethesda, MD, USA). The $P$. multocida strains were aerobically cultured in Martin's broth medium (Qingdao Hope Biol-Technology Co., Ltd., Qingdao, China) at $37^{\circ} \mathrm{C}$ for 12 h. E. coli $\mathrm{DH} 5 \alpha$ was aerobically cultured in Luria Bertani broth (LB; Sigma-Aldrich; Merck KGaA, Darmstadt, Germany) at $37^{\circ} \mathrm{C}$ for $20 \mathrm{~h}$. The $E$. coli strain genomic DNA was used as a negative control.

Mice and housing conditions. A total of 120 Female Kunming (KM) mice (8 weeks-old, body weight 18-22 g) were obtained from the Chongqing Academy of Chinese Materia Medica (Chongqing, China). The animals were housed in pathogen-free conditions (temperature, $20-30^{\circ} \mathrm{C}$; relative humidity, $45-60 \%$; 12-h light/dark cycle) and were given free access to food (standard rodent diet) and drinking water.

OMP preparation. OMPs were prepared in accordance with previously described protocols (19-21). The bacteria cell cultures were harvested by centrifugation at $10,000 \mathrm{x} \mathrm{g}$ for $15 \mathrm{~min}$ at $4^{\circ} \mathrm{C}$. The pellets were washed 3 times with cold, sterile PBS (pH 7.4) and resuspended in PBS ( $\mathrm{pH} 7.4)$ containing $8 \%$ Triton X-114, 0.5\% 3-(3-cholamidopropyl)-dimethylammo nio]-1-propanesulfonate and $1 \mathrm{mmol} / 1$ phenylmethylsulfonyl fluoride, and maintained at $4^{\circ} \mathrm{C}$ for $3 \mathrm{~h}$. The cell debris was removed via centrifugation at $10,000 \mathrm{xg}$ and $4^{\circ} \mathrm{C}$ for $10 \mathrm{~min}$, and the aqueous phase was collected and $10 \mu \mathrm{g}$ OMPs were detected by using $12 \%$ SDS-PAGE gel. The gelatinous decontamination phase was added into 1:9 volume absolute ethanol, and the pellets were collected by centrifugation at $10,000 \times \mathrm{g}$ and $4^{\circ} \mathrm{C}$ for $30 \mathrm{~min}$, washed twice with PBS, and dissolved into PBS. Concentration of OMPs was determined using the Bradford method (Bradford Protein Assay kit; Bio-Rad Laboratories, Inc., Hercules, CA, USA).
Western blot analysis and linear trap quadrupole (LTQ)/mass spectrometry $(M S)$. The $10 \mu \mathrm{g}$ OMPs were separated by using $12 \%$ SDS-PAGE gel and blotted electrophoretically onto a polyvinylidene difluoride membrane. The membrane was incubated with infected PmCQ2 bovine serum (PmCQ2-IS; 1:16) and horseradish peroxidase-conjugated rabbit anti-bovine IgG antibodies (cat. no. SAB3700018; 1:20,000; Sigma-Aldrich; Merck KGaA). An Enhanced Chemiluminescent reagent (Pierce; Thermo Fisher Scientific, Inc., Waltham, MA, USA) was applied for a chromogenic reaction. Proteins were visualized using a LTQ Mass Spectrometer (Shanghai Applied Protein Technology Co. Ltd., Shanghai, China). Proteins in P. multocida species were detected using BioWorks Browser software version 3.3 (bioworks.software.informer.com).

Protein expression and purification. The target genes were amplified from the genomic DNA of strain PmCQ2. DNApol was from Takara Biotechnology Co., Ltd. (Chongqing, China) and the primers for the selected genes are presented in Table I. The products of polymerase chain reaction $\left(94^{\circ} \mathrm{C}\right.$ for $3 \mathrm{~min}$, $94^{\circ} \mathrm{C}$ for $1 \mathrm{~min}, 50^{\circ} \mathrm{C}$ for $1 \mathrm{~min}, 72^{\circ} \mathrm{C}$ for $1 \mathrm{~min}, 35$ cycles at $72^{\circ} \mathrm{C}$ for $10 \mathrm{~min}$, stored at $4^{\circ} \mathrm{C}$ ) were cloned into pET-28a plasmid (Takara Biotechnology Co., Ltd.) in E.coli DH5a (Takara Biotechnology Co., Ltd.), expressed in E. coli BL21 (DE3; Takara Biotechnology Co., Ltd.), cultured at $37^{\circ} \mathrm{C}$ in LB broth or on agar supplemented with $100 \mu \mathrm{g} / \mathrm{ml} \mathrm{kanamycin}$ (Sigma-Aldrich; Merck KGaA) and induced with $0.5 \mathrm{mM}$ isopropyl $\beta$-D-1-thiogalactopyranoside (Sigma-Aldrich; Merck $\mathrm{KGaA}$ ) at $30^{\circ} \mathrm{C}$ or $37^{\circ} \mathrm{C}$ for $4 \mathrm{~h}$. The recombinant proteins were purified using affinity chromatography and Ni-NTA Superflow cartridges (Qiagen $\mathrm{GmbH}$, Hilden, Germany).

Anti-serum preparation. A total of $4 P$. multocida-antibody-free calves were purchased from a farm in Chongqing. One calf was immunized intramuscularly in the neck with $4.8 \times 10^{10}$ colony forming unit (CFU)-inactivated PmCQ2, which was fully emulsified with an equal volume of Freund's incomplete adjuvant (Sigma-Aldrich; Merck KGaA) following 3 administrations with 10-day intervals between each. The blood was collected for anti-serum (PmCQ2-VS) separation. A second calf was intramuscularly injected in the neck with $4.8 \times 10^{10} \mathrm{CFU}$ live PmCQ2. Following 3 injections with the same dose at 10-day intervals for each dose, the antiserums (PmCQ2-IS) obtained from the calf were separated post-infection at 30 days. The remaining two calves were intranasally infected with $4.8 \times 10^{10} \mathrm{CFU}$ live $\mathrm{PmB}$ and PmF 3 times. The calves were injected 3 times with the same dose, at 10 day intervals for each dose. Subsequently, the antiserums (PmB-IS and PmF-IS) were separated post-infection at 30 days.

Indirect ELISA. Recombinant protein was dissolved in coating buffer (0.5 M carbonate buffer; $\mathrm{pH} 9.6)$ and added to the well (10 $\mu \mathrm{g}$ protein/well) of 96-well microplates (Nunc-Immuno ${ }^{\mathrm{TM}}$ MicroWell; Merck KGaA) and then incubated at $4^{\circ} \mathrm{C}$ overnight. Plates were washed three times with PBS containing $0.05 \%$ Tween 20 (PBST), blocked for prevention of non-specific binding with $200 \mu 1$ 2\% skimmed milk (Sigma-Aldrich; Merck KGaA) at $37^{\circ} \mathrm{C}$ for $1 \mathrm{~h}$. A 1:100 dilution of bovine serum in blocking buffer was added to each well and incubated at $37^{\circ} \mathrm{C}$ for $1 \mathrm{~h}$. Following washing three times each 
Table I. Primer sequences for the selected genes.

\begin{tabular}{|c|c|c|c|}
\hline Gene & Forward $\left(5^{\prime}-3^{\prime}\right)$ & Reverse $\left(5^{\prime}-3^{\prime}\right)$ & Length \\
\hline$P m C Q 2 \_3 g 0306$ & CCGGAATTCGCACCACAACCTAAC & CCGCTCGAGTTATTTGTTACCTTTAACAGCG & 962 \\
\hline PmCQ2_2g0128 & $\begin{array}{l}\text { CCGGAATTCGAAAGTAAACA } \\
\text { GCAAGC }\end{array}$ & $\begin{array}{l}\text { CCGCTCGAGTTATTTCGCTAAGAGATTTTG } \\
\text { TAATGC }\end{array}$ & 951 \\
\hline PmCQ2_1g0327 & CCGGAATTCGGTGGGGGTAATAAC & $\begin{array}{l}\text { CCGCTCGAGTTATTGTTTTTTCTCCATACCTA } \\
\text { AAACACCCC }\end{array}$ & 948 \\
\hline$P m C Q 2 \_3 g 0121$ & CCGGAATTCCGTGCACCAAGTAGC & CCGCTCGAGTTAATCCGCTAACTTAGTGC & 1,500 \\
\hline$P m C Q 2 \_2 g 0046$ & CCGGAATTCGTTGAGCTCGCAAAAC & CCGCTCGAGTTAAATGCGTGATTCG & 975 \\
\hline$P m C Q 2 \_1 g 0020$ & CCGGAATTCGGCACCGAATTAGCC & CCGCTCGAGGAAGTTCTTACCAGGAT & 1,518 \\
\hline
\end{tabular}

Underlined bases represent the double enzyme loci of EcoRI/XhoI.

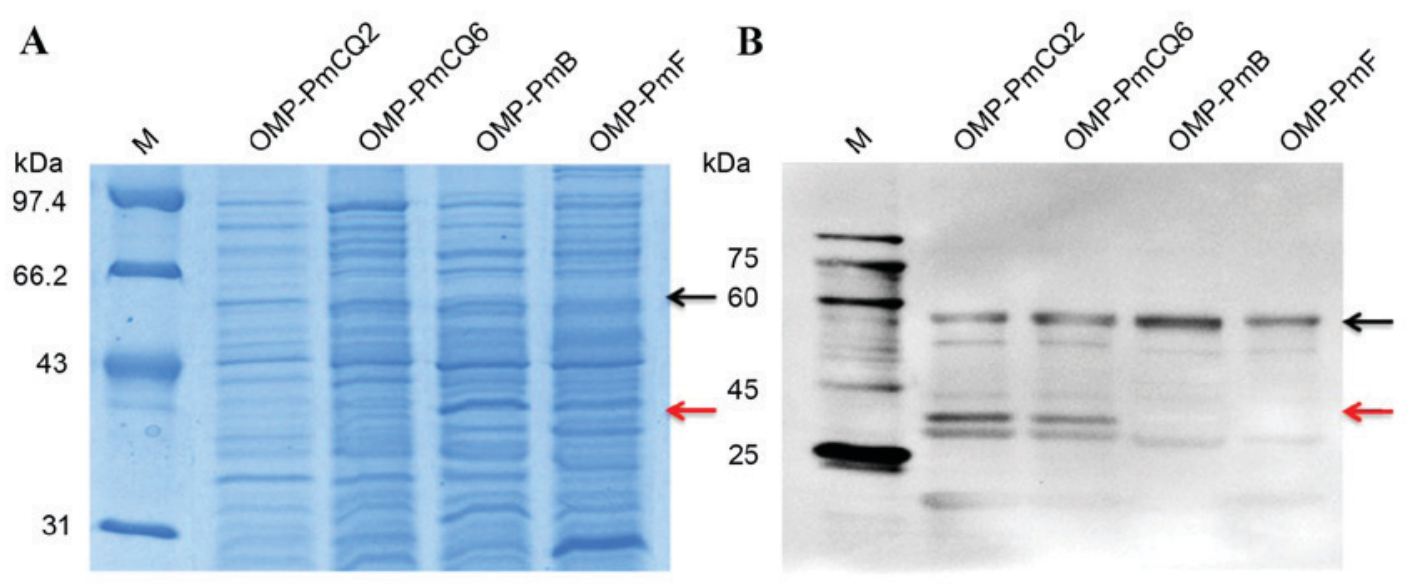

Figure 1. OMPs of P. multocida serotypes A, B and F. (A) SDS-PAGE of the extracted OMPs fragments. (B) Representative western blot images for OMP expression. Black arrows indicate the common band in P. multocida serotypes A, B and F. Red arrows indicated a unique band in $P$. multocida serotype A. OMP, outer membrane protein; M, marker; P. multocida, bovine Pasteurella multocida.

for 20 min with PBST, a 1:20,000 dilution of horseradish peroxidase-conjugated rabbit anti-bovine $\mathrm{IgG}$ antibodies (SAB3700018; Sigma-Aldrich; Merck KGaA) in blocking buffer was added to each well and incubated continuously at $37^{\circ} \mathrm{C}$ for $1 \mathrm{~h}$, then washed three times each for $20 \mathrm{~min}$ with PBST. Then, $100 \mu \mathrm{l}$ substrate solution $30 \mathrm{mg}$ o-phenylenediaminedihydrochloride (Takara Biotechnology Co., Ltd.) in $100 \mathrm{ml}$ substrate buffer $\left(0.2 \mathrm{M} \mathrm{Na}_{2} \mathrm{HPO}_{4}-12 \mathrm{H}_{2} \mathrm{O} ; 0.1 \mathrm{MC}_{6} \mathrm{H}_{8} \mathrm{O}_{7}\right.$; $\mathrm{pH} 4.8)$ ] was added to each well and incubated without light for 15-30 min at room temperature. The reaction was stopped by adding $100 \mu 12 \mathrm{~N}$ sulfuric acid $\left(\mathrm{H}_{2} \mathrm{SO}_{4}\right.$; Sigma-Aldrich; Merck $\mathrm{KGaA}$ ) and absorbance values were recorded at a wave length $450 \mathrm{~nm}$ by ELISA reader (Spectrophotometer 1510, Thermo Fisher Scientific, Inc.).

Mice immunization and challenge treatments. A total of 120 female KM mice were equally divided into 12 groups ( $n=10 /$ group). Each mouse was subcutaneously inoculated with the $100 \mu \mathrm{g} /$ dose recombinant proteins. The control group mice were inoculated with $100 \mu \mathrm{l}$ PBS. The initial two inoculations occurred once in two consecutive weeks and the third and final inoculation occurred in week 4. The mice were intramuscularly injected with $10 \mathrm{LD}_{50} \mathrm{PmCQ} 2\left(\mathrm{LD}_{50}=6.48 \times 10^{6} \mathrm{CFU}\right)$,
$\operatorname{PmB}\left(\mathrm{LD}_{50}=3.87 \times 10^{6} \mathrm{CFU}\right)$ and $\mathrm{PmF}\left(\mathrm{LD}_{50}=1 \times 10^{7} \mathrm{CFU}\right)$ strains 2 weeks after the third and final inoculation.

Statistical analysis. Data were analyzed by Microsoft Office Excel 2010 and SPSS version 20 (IBM SPSS, Armonk, NY, USA). Sequence homology analysis was employed using ClustalW2 (http://www.ebi.ac.uk/tools/clustalw2). Survival rates of animals were evaluated using Kaplan-Meier analysis and Life test of SAS 9.2 (SAS Institute Inc.) $\mathrm{P}<0.05$ was considered to indicate a statistically significant difference.

\section{Results}

OMP extraction and western blot analysis. The OMPs were extracted using Triton X-114 from P. multocida serotype A, $\mathrm{B}$ and $\mathrm{F}$ strains. The OMPs were identified by SDS-PAGE (Fig. 1A) western blot analysis (Fig. 1B) of PmCQ2-IS serum. A $~ 57 \mathrm{kDa}$ common OMP band was observed in PmCQ2, PmCQ6, PmB and PmF (indicated by black arrows in Fig. 1), and a clear $34 \mathrm{kDa}$ OMP band in PmCQ2 and PmCQ6 (indicated by red arrows in Fig. 1). To identify the candidate cross-protective antigens for $P$. multocida serotype A, the two gel bands were cut out and used for LTQ/MS analyses. 

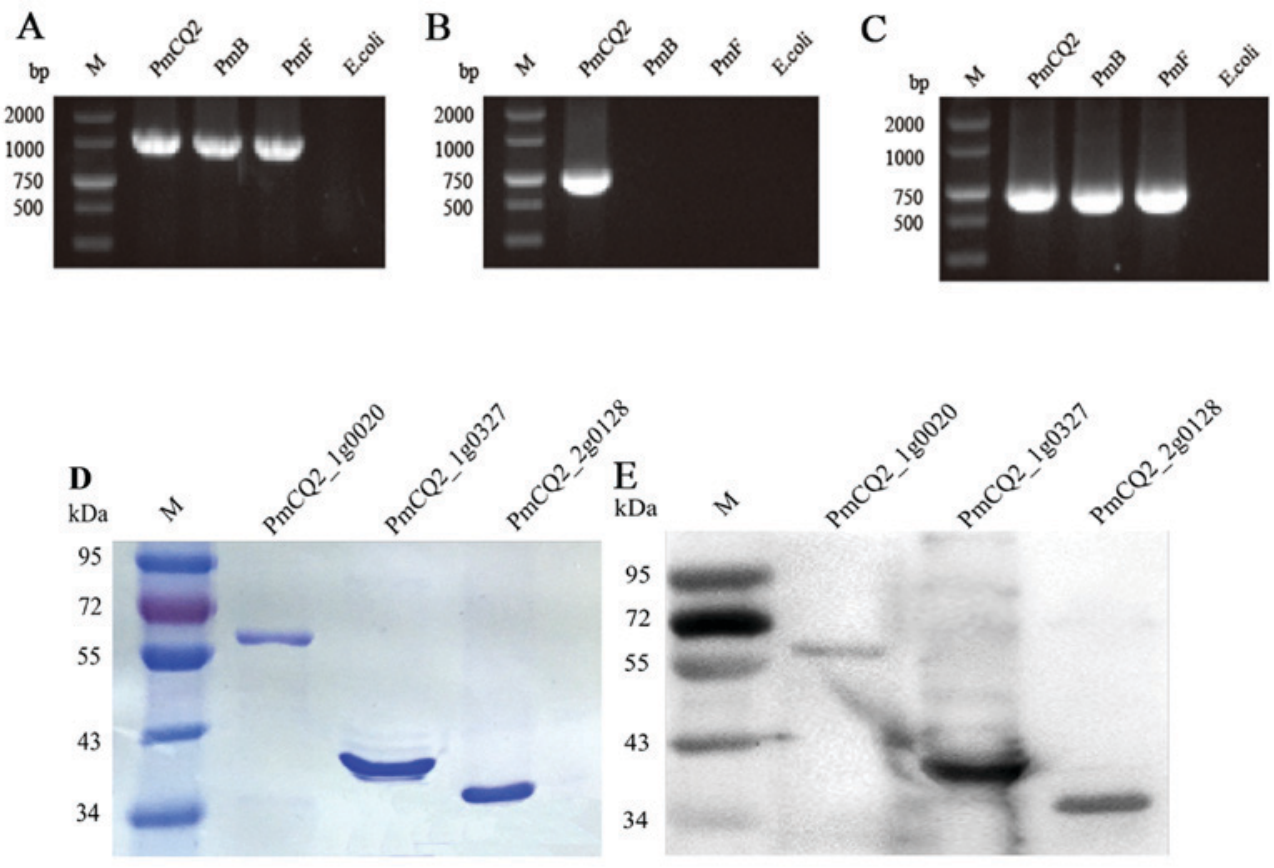

Figure 2. PCR amplification and purification of recombinant proteins. A 1.0\% agarose gel showing PCR-amplified gene products of (A) PmCQ2_2g0128, (B) PmCQ2_1g0327 and (C) PmCQ2_1g0020 gene products of PmCQ2. (D) Purified 3 recombinant protein fragments. (E) Representative western blot image of polyclonal sera (PmCQ2-IS). PCR, polymerase chain reaction; M, DL2000 DNA marker.

LTQ/MS and bioinformatics analysis. Based on the LTQ/MS analysis, the protein sequences were compared with the Uniprot database (www.uniprot.org) and our bovine P. multocida genome database. A total of 6 protein sequences were matched and are presented in Table II. These protein sequences were predicted as secretory proteins using TMHMM Server.2.0 (22), SignalPv3.0 (23), PSORTb (24) and GPI-SOM (25). A total of 6 protein sequences contained a signal peptide, and 3 secretory proteins were predicted from the bioinformatics analysis (Table I). Using the aforementioned combined analyses, 6 protein sequences were cloned and purified.

Recombinant protein selection. The sera for PmCQ2-VS, PmCQ2-IS, PmB-IS and PmF-IS were used to test the reactivities of 6 recombinant proteins using an indirect ELISA (Table III). rPmCQ2_3g0306, rPmCQ2_2g0128, rPmCQ2_3g0121 and rPmCQ2_2g0046 had the high reactogenicity to PmCQ2-IS; however, only rPmCQ2_1g0327 and rPmCQ2_1g0020 were able to react with the 4 sera. Therefore, the present study used r-PmCQ2_2g0128,r-PmCQ2_1g0327 and r-PmCQ2_1g0020 to verify potential cross-protective antigens and diagnostic antigens for P. multocida serotype A.

Characterization and protective efficacy of recombinant proteins. The present study extracted genomic DNA from the PmCQ2, PmB, PmF and E. coli strains to amplify recombinant proteins (Fig. 2). PmCQ2_1g0020 and PmCQ2_2g0128 DNA were amplified in PmCQ2, PmB and PmF (Fig. 2A and C, respectively). However, $P m C Q 2 \_1 g 0327$ DNA was not amplified in PmB and PmF (Fig. 2B). The purity of recombinant proteins was determined using SDS-PAGE, which revealed single bands with the molecular masses 34,35 and $57 \mathrm{kDa}$ (Fig. 2D). The identity and integrity of the recombinant proteins
(rPmCQ2_1g0020, rPmCQ2_1g0327 and rPmCQ2_2g0128) were determined using western blotting (Fig. 2E).

The homology analysis was performed with Clustal X software version 2.0 (http://www.ebi.ac.uk/tools/clustalw2) on PmCQ2_1g0327 and different P. multocida strains that contained PmCQ2, PmCQ6, PmB, PmF, 5 additional bovine $P$. multocida (isolated from the lungs of diseased cattle in our laboratory), 1 poultry $P$. multocida (isolated from the lungs of diseased anseriformes in our laboratory) and 27 additional $P$. multocida genome sequences from the National Center for Biotechnology Information (www.ncbi.nlm.nih.gov). Sequence analysis revealed that $P m C Q 2 \_1 g 0327$ exhibited a $100 \%$ sequence match to $P m u_{-} 17770$ and PmCQ6_c4301g0004 in P. multocida serotype A Pm36950 and PmCQ6 strains (Fig. 3).

In order to evaluate the protective efficacy of these proteins, mice were immunized with $100 \mu \mathrm{g} / \mathrm{dose}$ purified r-PmCQ2_2g0128, r-PmCQ2_1g0327 and r-PmCQ2_1g0020. Therefore, it was determined that up to 7 days after a challenge with PmCQ2, r-PmCQ2_1g0327 and r-PmCQ2_2g0128 proteins provided improved survival rates (50 and 40\%, respectively) compared with PBS alone (control group) and r-PmCQ2_1g0020 (0\%). No statistically significant difference was identified between the protection percentages (30-40\%) of these three proteins against PmB. However, the protection percentages against PmF were low or zero (Fig. 4).

\section{Discussion}

Pasteurella is a major respiratory infection in farm and wild animals that has various symptoms, such as swine plague, bovine pneumonia, hemorrhagic septicemia in cattle and abscesses, otitis, and septicemia in rabbit, cholera fowl and 
Table II. Linear trap quadrupole mass spectrometry and bioinformatics analysis.

\begin{tabular}{lcccccl}
\hline Protein & $\begin{array}{c}\text { Common } \\
\text { band }\end{array}$ & $\begin{array}{c}\text { Unique } \\
\text { band }\end{array}$ & $\begin{array}{c}\text { Signal } \\
\text { peptides }\end{array}$ & $\begin{array}{c}\text { Transmembrane } \\
\text { domains }\end{array}$ & $\begin{array}{c}\text { Secretory } \\
\text { protein }\end{array}$ & Description \\
\hline PmCQ2_3g0306 & 2 & 222 & $\mathrm{Y}$ & $\mathrm{N}$ & $\mathrm{Y}$ & Outer membrane protein A \\
PmCQ2_2g0128 & 0 & 111 & $\mathrm{Y}$ & $\mathrm{N}$ & $\mathrm{N}$ & $\begin{array}{l}\text { Outer membrane protein assembly } \\
\text { factor BamC }\end{array}$ \\
PmCQ2_1g0327 & 0 & 31 & $\mathrm{Y}$ & $\mathrm{N}$ & $\mathrm{N}$ & Putative transmembrane protein \\
PmCQ2_3g0121 & 10 & 0 & $\mathrm{Y}$ & $\mathrm{N}$ & $\mathrm{Y}$ & Heme-binding protein A \\
PmCQ2_2g0046 & 0 & 7 & $\mathrm{Y}$ & $\mathrm{N}$ & $\mathrm{N}$ & Glycosyl transferase,family 2 protein \\
PmCQ2_1g0020 & 7 & 0 & $\mathrm{Y}$ & $\mathrm{N}$ & $\mathrm{Y}$ & Peptide ABC transporter substrate- \\
& & & & & & binding protein
\end{tabular}

Common band is $\sim 58 \mathrm{kDa}$ band in three serotype A, B, F strains, the unique band is $\sim 35 \mathrm{kDa}$ band which was only in the $P$. multocida serotype. The value in table represents the extent of the match peptides. Y, yes; N, no.

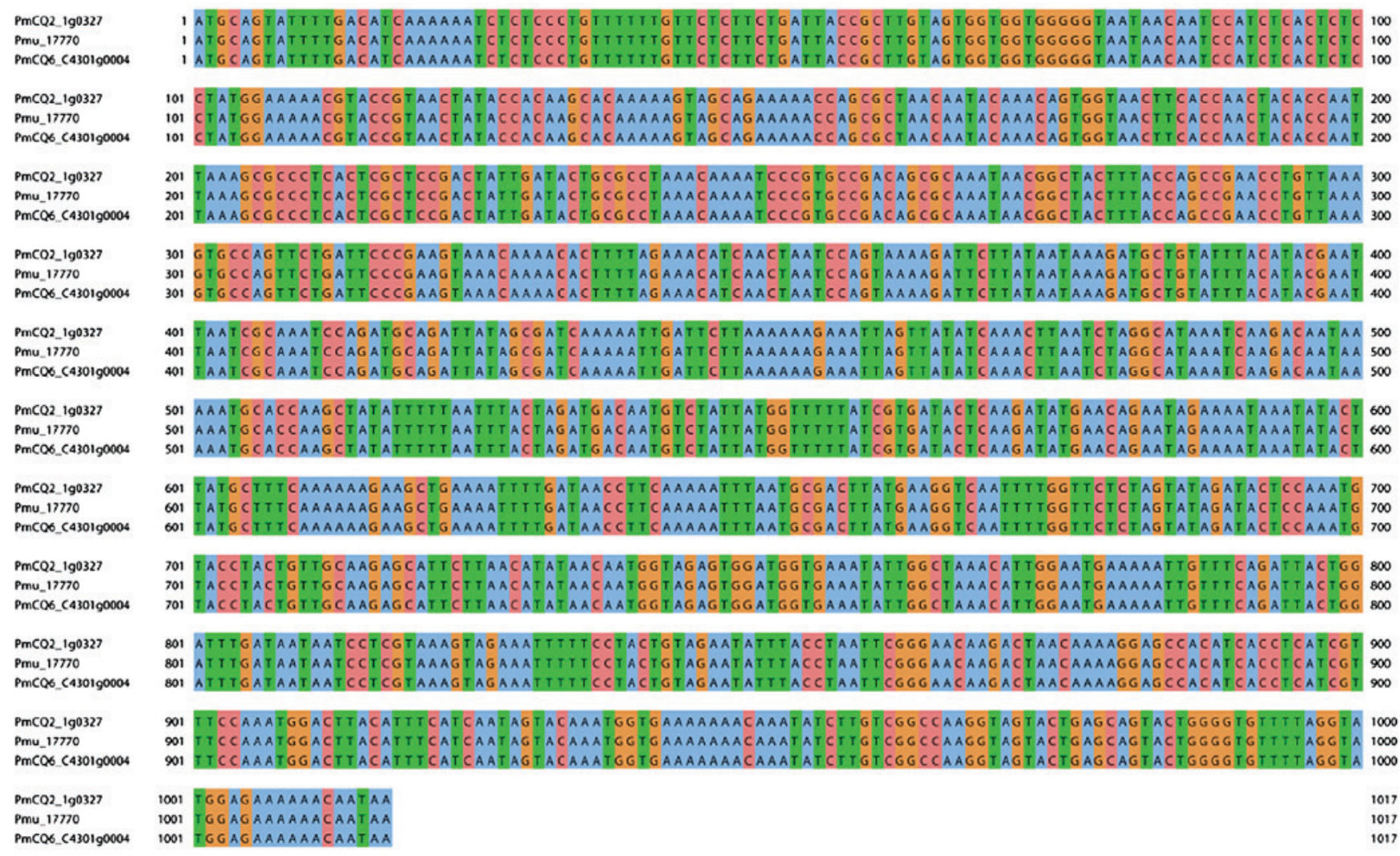

Figure 3. Sequence homology analysis for PmCQ2 (PmCQ2_1g0327), 36950 (Pmu_17770) and PmCQ6 (PmCQ6_C4301g0004). The bovine Pasteurella multocida serotype A strains PmCQ2 and PmCQ6 were isolated from the lungs of diseased cattle. The bovine respiratory disease isolate 36950 (GenBank: NC_016808.1) was downloaded from the NCBI.

swine atrophic rhinitis (1-4). P. multocida is the opportunistic pathogen responsible for this contagious disease. At present, commercially available vaccines are live attenuated vaccines and bacterin vaccines. The bacterins of $P$. multocida provided limited protection to heterologous serotypes and the live or attenuated vaccines may reverse the virulence of the animal disease. Based on the inevitable disadvantages, it is challenging to develop novel vaccine formulations which are effective, safe, have low production costs, and practicality for herd health (26). Bacterial OMPs are highly immunogenic and expose epitopes in the P. multocida envelope, which are important for conferring protective immunity in a range of infection models. Previous studies determined that the avian $P$. multocida $\mathrm{PlpB}$ protein was a cross-protective antigen (27), and this protein was located in the $P$. multocida outer membrane (28). The present study extracted OMPs from the different bovine $P$. multocida strains. Two protein bands were identified (30-40 kDa special bands 
Table III. Positive to negative value $(\mathrm{P} / \mathrm{N})$ ratios of the 6 recombinant proteins in the indirect ELISA.

\begin{tabular}{lcccr}
\hline P/N & $\begin{array}{c}\text { PmCQ2- } \\
\text { IS }\end{array}$ & $\begin{array}{c}\text { PmCQ2- } \\
\text { VS }\end{array}$ & $\begin{array}{c}\text { PmB- } \\
\text { IS }\end{array}$ & $\begin{array}{c}\text { PmF- } \\
\text { IS }\end{array}$ \\
\hline rPmCQ2_3g0306 & 5.53 & 1.45 & 2.50 & 1.98 \\
rPmCQ2_2g0128 & 6.97 & 1.87 & 2.11 & 1.56 \\
rPmCQ2_1g0327 & 8.22 & 7.68 & 8.71 & 10.06 \\
rPmCQ2_3g0121 & 3.36 & 1.83 & 0.89 & 1.12 \\
rPmCQ2_2g0046 & 5.35 & 1.47 & 1.35 & 1.29 \\
rPmCQ2_1g0020 & 6.92 & 6.05 & 6.28 & 8.52 \\
\hline
\end{tabular}

and 50-60 kDa common bands) by western blot analysis of PmCQ2-infected serum. Based on the LTQ/MS analyses, 6 proteins were cloned, expressed, purified and used for an indirect ELISA. Based on the $\mathrm{P} / \mathrm{N}$ values, it is possible that r-PmCQ2_3g0306, r-PmCQ2_2g0128, r-PmCQ2_3g0121 and r-PmCQ2_2g0046 represent potential diagnostic antigens for the P. multocida serotype A, based on high $\mathrm{P} / \mathrm{N}$ values with PmCQ2-IS. The r-PmCQ2_1g0327 and r-PmCQ2_1g0020 antigens may be potential cross-protective antigens due to the high $\mathrm{P} / \mathrm{N}$ values for the different serotypes A, B and F. Subsequently, 3 proteins were selected for evaluation of their protective efficacy in mice. The findings of the present study revealed that the r-PmCQ2_1g0327 and r-PmCQ2_2g0128 exhibited 40-50\% survival rates and may induce cross-immunity in mice against the P. multocida serotype A and B strains. The homology analysis revealed that $P m C Q 2 \_1 g 0327$ exhibited $100 \%$ sequence identity with three $P$. multocida serotype A strains; therefore, $P m C Q 2 \_1 g 0327$ may be used as a diagnostic antigen for $P$. multocida serotype A strains. However, the function of this protein requires further investigation.

B-cell epitopes have an important role in vaccine development and disease diagnosis (29,30). Epitope identification provides valuable information that is essential for understanding antigen components (31). The present study did not identify PmCQ2_lg0327 in the PmB and PmF genomes; however, r-PmCQ2_1g0327 protected against a PmB infection challenge. Therefore, the present study identified the B-cell epitopes for associated genes in PmCQ2 and PmB using the Protean software and the DNAStar package. The findings revealed specific antigenic epitopes, which were similar to r-PmCQ2_1g0327 in PmCQ2 and PmB.

In conclusion, r-PmCQ2_1g0327 and r-PmCQ2_1g0020 may serve as cross-protective antigens or effective vaccine components against infection with P. multocida serotypes A and B. However, the two recombinant proteins require additional investigation to verify protective immunity in cattle and confirm potential cross-protective antigens to develop P. multocida vaccines. In addition, the protective immunity of the residual proteins (r-PmCQ2_3g0306, r-PmCQ2_3g0121 and r-PmCQ2_2g0046) require investigation, as they exhibit higher OD values with the live PmCQ2 infected serum and not the inactivated PmCQ2 immune serum. Therefore, these proteins may be serve as diagnostic antigens for P. multocida serotype A strains. Additionally, the ELISA assay platform
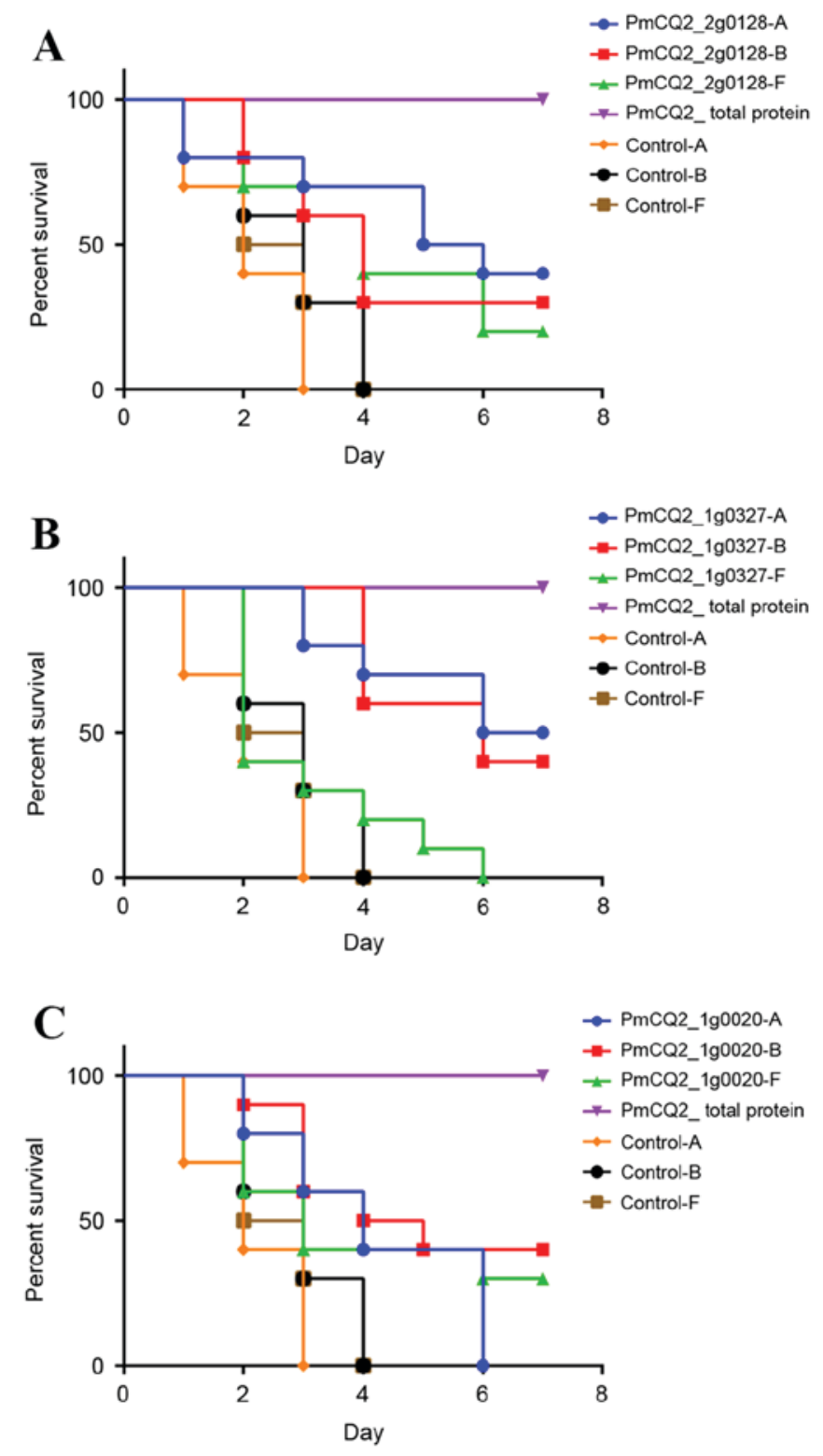

Figure 4. Protective efficacy of the recombinant proteins in mice model. (A) r-PmCQ2_2g0128, (B) r-PmCQ2_1g0327 and (C) r-PmCQ2_1g0020 were used to immunize the mice at a dosage of $100 \mu \mathrm{g} /$ mice. The percentage survival curve revealed the survival/mortality pattern for immunized and control mice (10 mice/group) following a challenge with $10 \mathrm{LD}_{50}$ PmCQ2, $\mathrm{PmB}$ and PmF. PmCQ2_total protein was used as a positive control.

with specific sera (three serotypes) was a useful tool for the selection of protective and diagnostic antigens.

A previous study suggested that identifying OMPs may be important in the development of novel vaccine candidates and diagnostic antigens (32). Therefore, future studies should focus on the development of novel vaccines for P. multocida strains, and the generation of fusion proteins that may provide improved protection against different $P$. multocida strains.

\section{Acknowledgements}

The present study was supported by the China Agriculture Research System (Beef/Yak Cattle; grant no. CARS-38), the Chongqing Science \& Technology Commission (grant nos. cstc2015shmszx80022 and cstc2015jcyjBX0108), the National 
Natural Science Foundation of China (grant no. 31400762), the Fundamental Research Funds for the Central Universities (grant no. XDJK2015B002) and the Doctoral Funds of Southwest University (grant nos. SWU112006 and SWU114032).

\section{References}

1. Dagleish MP, Hodgson JC, Ataei S, Finucane A, Finlayson J, Sales J, Parton R and Coote JG: Safety and protective efficacy of intramuscular vaccination with a live aroA derivative of Pasteurella multocida B:2 against experimental hemorrhagic septicemia in calves. Infect Immun 75: 5837-5844, 2007.

2. Blackall PJ, Fegan N, Pahoff JL, Storie GJ, McIntosh GB, Cameron RD, O'Boyle D, Frost AJ, Bará MR, Marr G and Holder J: The molecular epidemiology of four outbreaks of porcine pasteurellosis. Vet Microbiol 72: 111-120, 2000.

3. Palócz O, Gál J, Clayton P, Dinya Z, Somogyi Z, Juhász C and Csikó G: Alternative treatment of serious and mild Pasteurella multocida infection in New Zealand White rabbits. BMC Vet Res 10: 276, 2014.

4. Pedersen K, Dietz HH, Jørgensen JC, Christensen TK, Bregnballe T, Andersen TH: Pasteurella multocida from outbreaks of avian cholera in wild and captive birds in Denmark. J Wildl Dis 39: 808-816, 2003.

5. Carter GR: Serological classification of Pasteurella multocida. Vet Rec 121: 382-383, 1987.

6. Carter GR: Studies on Pasteurella multocida. I. A hemagglutination test for the identification of serological types. Am J Vet Res 16: 481-484, 1955.

7. Praveena PE, Periasamy S, Kumar AA and Singh N: Pathology of experimental infection by Pasteurella multocida serotype A: 1 in buffalo calves. Vet Pathol 51: 1109-1112, 2014

8. Shivachandra SB, Viswas KN and Kumar AA: A review of hemorrhagic septicemia in cattle and buffalo. Anim Health Res Rev 12: 67-82, 2011.

9. Jaglic Z, Jeklova E, Christensen H, Leva L, Register K, Kummer V, Kucerova Z, Faldyna M, Maskova J and Nedbalcova K: Host response in rabbits to infection with Pasteurella multocida serogroup F strains originating from fowl cholera. Can J Vet Res 75: 200-208, 2011

10. Kumar AA, Shivachandra SB, Biswas A, Singh VP, Singh VP and Srivastava SK: Prevalent serotypes of Pasteurella multocida isolated from different animal and avian species in India. Vet Res Commun 28: 657-667,2004.

11. Ahmad TA, Rammah SS, Sheweita SA, Haroun $M$ and El-Sayed LH: Development of immunization trials against Pasteurella multocida. Vaccine 32: 909-917, 2014.

12. Singh AP, Singh S, Ranjan R, Gupta SK, Singh VP and Sharma B: Molecular heterogeneity of plpE gene in Indian isolates of Pasteurella multocida and expression of recombinant PlpE in vaccine strain of P. multocida serotype B: 2 . J Vet Sci 11: 227-233, 2010.

13. Lin J, Huang S and Zhang Q: Outer membrane proteins: Key players for bacterial adaptation in host niches. Microbes Infect 4: 325-331, 2002.

14. Wu JR, Shien JH, Shieh HK, Chen CF and Chang PC: Protective immunity conferred by recombinant Pasteurella multocida lipoprotein E (PlpE). Vaccine 25: 4140-4148, 2007.

15. Sthitmatee N, Numee S, Kawamoto E, Sasaki H, Yamashita K, Takahashi N, Kataoka Y and Sawada T: Protection of chickens from fowl cholera by vaccination with recombinant adhesive protein of Pasteurella multocida. Vaccine 26: 2398-2407, 2008.
16. Confer AW, Suckow MA, Montelongo M, Dabo SM, Miloscio LJ, Gillespie AJ and Meredith GL: Intranasal vaccination of rabbits with Pasteurella multocida A:3 outer membranes that express iron-regulated proteins. Am J Vet Res 62: 697-703, 2001.

17. Harada T, Sakakura Y and Miyoshi Y: Immunological response to outer membrane vesicles of Haemophilus influenzae in patients with acute sinusitis. Rhinology 24: 61-66, 1986.

18. Prado ME, Dabo SM and Confer AW: Immunogenicity of iron-regulated outer membrane proteins of Pasteurella multocida A:3 in cattle: Molecular characterization of the immunodominant heme acquisition system receptor (HasR) protein. Vet Microbiol 105: 269-280, 2005.

19. Bordier C: Phase separation of integral membrane proteins in Triton X-114 solution. J Biol Chem 256: 1604-1607, 1981.

20. Rimler RB: Purification of a cross-protective antigen from Pasteurella multocida grown in vitro and in vivo. Avian Dis 45: 572-580, 2001.

21. Rimler RB and Rhoades KR: Lysates of turkey-grown Pasteurella multocida: Protection against homologous and heterologous serotype challenge exposures. Am J Vet Res 42: 2117-2121, 1981.

22. Chen Y, Yu P, Luo J and Jiang Y: Secreted protein prediction system combining CJ-SPHMM, TMHMM, and PSORT. Mamm Genome 14: 859-865, 2003

23. Bendtsen JD, Nielsen H, von Heijne G and Brunak S: Improved prediction of signal peptides: SignalP 3.0. J Mol Biol 340: 783-795, 2004.

24. Yu NY, Wagner JR, Laird MR, Melli G, Rey S, Lo R, Dao P, Sahinalp SC, Ester M, Foster LJ and Brinkman FS: PSORTb 3.0: Improved protein subcellular localization prediction with refined localization subcategories and predictive capabilities for all prokaryotes. Bioinformatics 26: 1608-1615, 2010.

25. Fankhauser N and Mäser P: Identification of GPI anchor attachment signals by a Kohonen self-organizing map. Bioinformatics 21: 1846-1852, 2005.

26. Thanasarasakulpong A, Poolperm P, Tankaew P, Sawada T and Sthitmatee N: Protectivity conferred by immunization with intranasal recombinant outer membrane protein $\mathrm{H}$ from Pasteurella multocida serovar A:1 in chickens. J Vet Med Sci 77: 321-326, 2015.

27. Tabatabai LB and Zehr ES: Identification of five outer membrane-associated proteins among cross-protective factor proteins of Pasteurella multocida. Infect Immun 72: 1195-1198, 2004.

28. Boyce JD, Cullen PA, Nguyen V, Wilkie I and Adler B: Analysis of the Pasteurella multocida outer membrane sub-proteome and its response to the in vivo environment of the natural host. Proteomics 6: 870-3280, 2006.

29. Saha S and Raghava GP: Prediction of continuous B-cell epitopes in an antigen using recurrent neural network. Proteins 65: 40-48, 2006.

30. Sollner J, Grohmann R, Rapberger R, Perco P, Lukas A and Mayer B: Analysis and prediction of protective continuous B-cell epitopes on pathogen proteins. Immunome Res 4: 1, 2008.

31. Mishra A, Jain A and Arora N: Mapping B-cell epitopes of major and minor peanut allergens and identifying residues contributing to IgE binding. J Sci Food Agric 96: 539-547, 2016.

32. Prasannavadhana A, Kumar S, Thomas P, Sarangi LN, Gupta SK, Priyadarshini A, Nagaleekar VK and Singh VP: Outer membrane proteome analysis of Indian strain of Pasteurella multocida serotype B:2 by MALDI-TOF/MS analysis. ScientificWorldJournal 2014: 617034, 2014. 